\title{
Research on Influence of Contextualization on Difficulty of Test Questions
}

\author{
Fei Wang \\ Wenshan University, Wenshan, 663000, China
}

Keywords: Test questions, Contextualization, Item difficulty

\begin{abstract}
Under the background of quality teaching, teaching evaluation should pay special attention to whether students can abstract knowledge points from actual situations and solve problems by using knowledge points in contextualization questions. The contextualization of the test question increases the amount of information, making the question more complicated and increasing the difficulty of the test questions. This paper analyzes the factors that influence the difficulty of contextualization question from question length, logical levels, coordinate units and language expression to provide some references for relevant researchers.
\end{abstract}

\section{Introduction}

The contextualization question is a question based on the situation. Since these questions are based on the material of real life, they have the characteristics of lively and open materials. Contextualization questions have been paid more and more attention in teaching, and it is an important tool to cultivate students' ability to use their knowledge and think independently. Contextualization questions are emphasized and applied in the examination, especially in the high-level selection examination, such as the college entrance examination. Contextualization examination questions try to build knowledge and real-life connections through the organization of background materials to show students what kind of knowledge they have learned in school and give meaning to learning. During knowledge learning in the school background, teaching is based on the knowledge unit. The traditional test questions are also centered on knowledge, and more of the simple examination of knowledge and skills, often disjointed with real life. During teaching and examination, students are simply told that the knowledge will be useful in the future. With the development of the economic and social and educational level, the use of knowledge has attracted more and more attention. Traditional knowledge-based examinations, such as examinations based on single knowledge, skills or knowledge, are increasingly showing their limitations. The examination from the memory of knowledge to the emphasis on the use of knowledge, and more emphasis on the ability to solve the problem of students. The change in the test goal determines the change of the test question design. The contextualization test is the basic means to realize the goal of knowledge application and problem-solving ability detection. The test situation is based on the material in real life. For students, it is often novel, and no ready-made answer can be applied. It requires students to overcome obstacles by mobilizing their knowledge and skills to solve the problem.

\section{Difficulty Comparison of Contextualization Questions and Non-Contextualization Questions}

The problems in real situations can make the abstract problem specific. Therefore, people mistakenly think that the contextualization test questions to become specific and easy to understand, so that the test of variation. However, the fact is that people underestimated the influence of contextualization questions on students' problem-solving performance, and the contextualization of test questions increased the amount of information and made the problem more complicated. If the examinee is not familiar with the situation, it will impede their understanding and solution to the problem, thus increasing the difficulty of the test. The situation of the test questions will affect the students' strategies to solve or explain the problems. The international large-scale education 
assessment has a strong sense of situation. Situated in the context of real scientific situations, information is presented according to the general path of academic research. Students need to find problems through understanding situations, and use existing knowledge reserves to build path to solve problems and apply them. For the same point of knowledge, we compare the difficulty of the contextualization and non-contextualization questions, which are shown in Table 1. We can clearly see that the difficulty of the contextualization test is obviously higher than the difficulty of the non-contextualization test question.

Table 1. Empirical research of difficulties of contextualization questions and non-contextualization questions

\begin{tabular}{|l|r|r|}
\hline & Contextualization Questions & Non-Contextualization Questions \\
\hline Question 1 & 0.53 & 0.82 \\
\hline Question 2 & 0.59 & 0.93 \\
\hline Question 3 & 0.69 & 0.75 \\
\hline Question 4 & 0.17 & 0.88 \\
\hline Question 5 & 0.42 & 0.89 \\
\hline Average difficulty & 0.48 & 0.85 \\
\hline
\end{tabular}

\section{Difficulty Comparison of Contextualization Questions of Difficult Types}

\subsection{Question Length}

On the premise of other factors, the longer the length of the text or the contents of the test questions, the greater the difficulty coefficient of the test questions. The contextualization level of the test questions has a significant influence on the difficulty level of the test questions. Contextualization questions that contains questions in the physical model is subtler, so that the increase of the difficulty of modeling test questions to the candidates. It contains more irrelevant information, thus increasing the difficulty of solving problems on students. For familiar situations, examinees are easy to be influenced by mindset, and directly use familiar models to solve problems, while ignoring the calm analysis of new situations, and ultimately lead to wrong answers to test questions. Therefore, for those situations whose surface characteristics are like those that students are familiar with, but the essential characteristics are quietly changing, the higher the familiarity of the surface features, the more difficult the questions are. The test paper based on multiple choice questions is very long, all of which are more than ten pages. But the examinee can complete or finish the answer in a more relaxed time in the prescribed time, which is determined by the nature and characteristics of the multiple-choice questions. If the examination paper is mainly used, the length of the examination paper can be reduced by more than half, because the examinee is much more intense when they are doing this kind of test. In this way, it looks short on the surface, but the validity of the examination paper is greatly improved. Natural work as the multiple-choice score is simple, but because the test space is greatly reduced, even under two, workload is not quite different, so it is feasible. In the curriculum test, the reading articles are all extracurricular contents. If the space is too large or the reading volume is too large, students can't finish the examination within the prescribed time, which is bound to affect the scores.

\subsection{Logical Levels}

Each of the Contextualization questions contains several logical levels. In general, the more contextualization questions, including the more logical level, the more difficult it is. The logical levels of different contextualization questions are often different. It is part of the contextualized test blanks, multiple-choice questions, short answer often involves only the memory of the students, if the students can remember the relevant content, according to the context, or think of the answer according to the problem of dry, without consideration of any other. In Chinese, fill in the blanks according to the content of the text, supplement the text content of the vacancy part, fill the vacancies 
in our historical figures, add up two figures in mathematics. Memory is also a kind of thinking at a certain level, but this thinking is very mechanical thinking, and even in a sense it is not thinking, but a simple representation in psychology. It is here as a kind of thinking problem that contains only one level. The so-called depth refers to the depth of the contextualization examination questions, which is one of the content factors that affect the difficulty of the contextualization test. Since the process of understanding people is from the shallower to the deeper, from the outside to the inside, from phenomenon to essence. Therefore, it is inevitable to master the superficial knowledge of the surface, and it is difficult to master the deep knowledge of the inner nature. Reflected in the situation test questions, the depth of knowledge changes will inevitably have an impact on the difficulty of the situation test questions. The present examination program is only a description of the scope of knowledge, and lacks the requirement for the depth of knowledge. It is necessary to organize some subject experts or propositional teachers to do some similar supplementary provisions carefully. The definition and direct description of nouns, principles and specific facts can be regarded as the first level of knowledge depth, and further inference and related nature theories are the second levels. The deep connotation and extension of knowledge and its internal relations with related knowledge, the nature and characteristics under special circumstances are the third levels. As the level of depth increases, the content of contextualization test examination is gradually deepened. In the absence of other factors, the difficulty will increase, and vice versa.

\subsection{Coordinate Units}

Some contextualization questions are made up of single meaning units. The content or questions of some questions are made up of several meaningful units. The test questions composed of several meaningful units are more difficult than those consisting of single meaning units. For example, materials for reading and analyzing questions, some questions are composed of a relatively simple meaning material, while some subjects have two independent material materials: "material one" and "material two". In other conditions, the latter is obviously more difficult than the difficulty of the former. Because the more meaning unit or component part of the test question, the more the content of the examinee to think about, the more difficult it is. The difficulty coefficient of the test question can be made by analogy, the more the meaning unit of the test question, the greater the degree of difficulty. A point of knowledge is an independent and complete minimum of knowledge, such as a certain concept, theorem, and specific facts listed in the examination outline. People grasp or understand knowledge from less to more, from easy to difficult to gradually accumulate. Accordingly, in specific propositions, teachers can make many levels of breadth. A single knowledge point is the first level. The two to three knowledge points should be second levels, and four or more knowledge points should be third levels. With the increase of the breadth level, the knowledge content of the contextualization test examination is rich. Therefore, in the case of non-consideration of other factors, the extent of the situation test question is increased, the difficulty of the contextualization test is improved, and vice versa. The division of knowledge points can be realized by the method of refining the outline. The refinement of the outline can determine the number of knowledge points of the contextualization test examination. In the proposition, the teacher can use the method of increasing the examination content to improve the difficulty of the situation test question. We can also reduce the difficulty by reducing the content of the assessment.

\subsection{Language Expression}

In contextualization questions, there are many key statements that are very precise and clear, but when students solve problems, they often forget the conditions of the questions, resulting in errors. Therefore, there is another key step to solve problems, which is to grasp the language in the topic and apply every precise expression to the full, to make it easier and easier to reduce errors. Contextualization questions are a part of the subject requires students to try to figure out the meaning, in solving the problems before figuring out the meaning of the title, the complex expressions into simple expressions, the semantics of transformation also has a key role on Contextualization questions to answer, to simplify the mathematical problem. There are many kinds of problems in 
contextualization test questions, but there are commonalities behind so many types of problems. It can be summed up as the abstraction, accuracy and complexity of contextualization question language. Students need to continue to explore the meaning of the title, carefully analyze the situation to be able to understand the meaning of the questions, we can find the solution. The language factor is the key to the problem of the situation test problem, and more pondering will find the breakthrough point. In the long-term learning, education and research practice, there are a certain percentage of questions or questions in exams for any subject and usual homework. Due to the statement errors in the statements, or the defects in the design of the test questions, the difficulty coefficient of such questions is also increased. This kind of situation is the error increase or abnormal enlargement of the test difficulty coefficient, which should be avoided in the proposition.

\section{Key Development Points of Contextualization Questions from the Perspective of Difficulty}

We should scientifically control the difficulty of information, the length of information and the density of information in the compilation of contextualization questions. Professional vocabulary, unfamiliar vocabulary, and even unnecessary long sentences, which are not related to students' learning, can cause understanding obstacles and weaken the achievement of the goal. Too long unnecessary information and high-density information will occupy students' working memory, which may make students ignore the main information and increase the difficulty of examination questions. The setting of the contextualization task is directly related to the examination of the target, whether the setting is reasonable, and whether the examination of the test question can be realized. The situation task is also the connection point of the test question material and the score design, which is in the key position in the whole test question design. Scientific setting of contextualization tasks should enable students to understand the intention of examination and make clear the direction and scope of answering. This is mainly achieved through the selection of instruction words and open control. The instruction words relate to the categories of the cognitive process to tell the students what to do. Through the instruction words, students can identify the categories of cognitive processes and how to use their ability to use knowledge. Whether students can hold their own perspectives and attitudes, can students solve problems in different ways of thinking and methods? The setting of the opening of the contextualization task also needs to consider the formulation of the scoring system. A conditional open question refers to a test subject to the scope of the answer, which is not only open, but also through the restrictive conditions of the perspective and knowledge. Due to some constraints, the boundary of students' answers is guided, which is good for scoring operation. A good balance between the openness of tasks and the operability of scores is achieved.

\section{Conclusions}

The purpose of setting up the situation test is to lead the students to learn to construct and apply knowledge and to cultivate students' scientific accomplishment. Compared with non-contextual test questions, situational test questions have higher requirements for candidates to analyze problems and solve problems, so it is more conducive to examine students' understanding and application ability of knowledge. In the compilation process of situational test questions, we should pay attention to the influence factors of the difficulty of the situational test questions, and control the difficulty of the situational test questions within a reasonable range.

\section{References}

[1] Zhang Junbing. Revelation from Xavier Roegiers' Thought about Designing Contextualization Questions [J]. China Examiniztaions, 2013(6): 26-32.

[2] Kong Yan, Wu Rumin, Zhu Xiaoguo, et al. Target Localization and Strategies of Developing Academic Context Items [J]. China Examiniztaions, 2016(9): 18-23.

[3] Du Mingrong. Influence of Contextualization on Difficulty of Test Questions [J]. China 
Examiniztaions, 2009(9): 40-44.

[4] Zhang Ying. Explore to analysis of questions that the creation contextualization Examination of high school mathematics [J]. 2010(3): 82. 